\title{
Prevalence of Permanent First Molar Loss in Intermediate Schools in Dammam Area, Saudi Arabia
}

\author{
Authors \\ Husam A.Alshamrani, Ghanim M.Alqahtani, Abdullah A.Alsani, \\ Abulrahman M. Alqahtani, Mohammad A. Hamidaddin, Hamdan M. Alotaibi
}

General Dental Practitioners

\begin{abstract}
Dental caries is an infectious disease that can start as soon as the first primary tooth erupts. In permanent teeth, it can begin around the age of 6 years with the eruption of first permanent molar. It is a serious problem, which may lead to loss of teeth as a consequence of neglected oral hygiene, poor diet control, lack of parents' education and monitoring in case of childhood caries. Also, prevalence of dental caries has been found to be higher in the low SES. The most common permanent teeth affected are molars as they are the first teeth to erupt. Molar teeth loss may have a negative impact on oral health, some of which include malocclusion, defect in mastication apparatus, complication on future treatments, and effect on self-esteem.

Keywords: Dental, Tooth-loss, Extraction, orthodontics
\end{abstract}

\section{INTRODUCTION}

Tooth loss has been reported in the literature with various numbers. In the United Kingdom (UK), a study was conducted in 2007 on a sample of 300 adolescents with a mean age of 11 years old where they found that $70 \%$ of the sample examined had dental caries. In a Brazilian study of 242 subjects, ranging in age from 14 to 82 years (mean 36.2 years), of the 200 subjects, $90 \%$ had lost at least one tooth, and $39 \%$ had lost more than 8 teeth. First mandibular molars were the most commonly missing teeth. In another study conducted in Nigeria at the Oral Surgery clinic of the University of Benin Teaching Hospital, it was found that the most frequently extracted tooth was the lower right first molar $(\mathrm{n}=109,10.4 \%)$ and the lower left first molar $(\mathrm{n}=95,9.0 \%)$, respectively. 157 molar extractions was done within the age group (14-20) years old female and male patients which represent $14.9 \%$ of the sample.

In A recent study that was done in turkey in 2013, the authors found 1615 tooth loss in a sample of 2925 subjects. Interestingly, $(n=48530 \%)$ of the tooth loss was within the age group of (13-15) years old. A study in Kuwait about the prevalence of first molar loss in 13-14 year-old school children that was conducted on a population-based sample of 1,583 eighth grade students. The investigators in this study found that there was $11.4 \%$ tooth loss prevalence. Almost $70 \%$ of this occurred only in the mandible, and the majority $(75.7 \%)$ had only one tooth affected. In a similar study in Riyadh, Saudi Arabia, it was found that $10.9 \%$ of missing teeth were mandibular first molars. The sample consisted of 1554 randomly chosen subjects. In the age group of $(10-20)$ years old, the missing teeth were $(n=81$ $5.2 \%)$. 
There seems to be a lack of literature regarding the prevalence of tooth loss in Saudi Arabia. Thus, the aim of this study was to assess the prevalence of permanent first molar loss in intermediate school students in the age group of (13-15 years old) and to identify the underlying causes.

\section{Materials and Methods}

In this cross-sectional study, the sample was chosen according to the General Administration of Education in the Eastern province database, where the total number of intermediate school students in Khobar and Dhahran was 4296. Using a confidence level of $95 \%$ and a $5 \%$ margin of error, the recommended sample size was 353 using Raosoft software. Therefore, our sample consisted of 350 students attending intermediate schools from different neighborhoods with different socioeconomic status (SES), King Fahad University School in Dhahran (High SES), Ibn alMudaffar School in Dhahran (Middle SES), and Ibn Majed intermediate governmental school in Khobar (Low SES).

The sample was visually examined for first permanent molar loss using flashlights and tongue depressors by two calibrated examiners. p, The calibration was done using 25 pictures of different cases of missing and decayed molars, and kappa of 0.93 was calculated using SPSS software (Statistical Package for the Social Sciences). The clinical criteria for assessment of missing molars in our study was any molar lost due to caries and was not seen in the mouth visually only as taking radiographs was not possible. As for clinical assessment of decayed molars, this was examined visually where in the case of no clinical crown visible or remaining roots this was considered grossly decayed and extraction was recommended. In addition to the clinical examination, each individual in the sample was asked to fill a survey at the time of examination and they were given another survey for their parents to answer in order to gain information about past dental health, oral hygiene measures practiced at home, and to ascertain if the tooth lost was permanent or not as participants may have congenitally missed teeth. The parents' surveys were collected the day after; as each school was visited into two successive days. Both surveys were structured in order to gather information about demographic and environmental variables, in addition to the subject perception toward his oral health, history of pain, reason of loss, and frequency of dental visits. Ethical approval was received from the scientific research committee at the College of Dentistrym University of Dammam

\section{Results}

There were equal weights of samples from each school. Of the 350 students about $50 \%$ of subjects brushed their teeth only once, $80 \%$ did not use dental floss. However 54\% thought they had fair oral hygiene. Almost $70 \%$ does not visit dentists regularly and $66 \%$ had previously experienced pain in their teeth. Regarding the feeling of students, $15 \%$ felt unhappy during dental visits. Students who had more than two snacks a day constituted $32 \%$ of the sample. Moreover, nearly 55\% of parents had higher education levels. As reported by them, $60 \%$ of the students consumed fluoridated water. $21.3 \%$ had permanent extractions, $83.6 \%$ of which were due to caries. $10.7 \%$ of the parents thought their children were behaving uncooperatively during dental treatment.

SES showed significant relation with tooth loss $(\mathrm{p}=0.000)$ and tooth decay $(\mathrm{p}=0.000)$. With subjects who had dental insurance, tooth loss $(p=0.003)$ and tooth decay $(\mathrm{p}=0.000)$ were significantly reduced. Parent education showed a level of significance with tooth loss $(p=0.045)$ where with tooth decay it was insignificant $(\mathrm{p}=0.333)$.

\section{Discussion}

This study was carried out to assess the prevalence and risk indicators of permanent first molar loss among intermediate school students in the age group (13-15) years old. Despite the fact that low numbers of molar loss was observed. The occurrence of tooth loss was significantly related with several variables, including socioeconomic 
status, dental insurance, and parent's education. For the best understanding of the underlying cause the prevalence and association were analyzed.

A sample of 350 adolescents showed a prevalence of 5.2\% molar loss, which is considered low compared to other studies. Atieh, 2008 reported in a study done on a sample of 484 , of the teeth lost the prevalence of first molar loss was $57.1 \%$. Similar to this study Atieh found that caries was the most common cause of tooth loss $(81.1 \%) .12$ Khalil et al., found $5.2 \%$ first molar loss, which is quiet similar to our study.9 In turkey Koray et al., he documented percentage of first molar loss was 6.3\%.7 Where in Kuwait Artun el al., reported a prevalence of $11.4 \%$ molar loss.

Although our sample was 350 the number with decayed permanent first molars that were indicated for extraction was relatively small and the number of missing permanent first molar was even smaller, when we examined SES with mean decayed and mean missing teeth we found that there is a significant relation. $(\mathrm{p}=0.000)$ and $(\mathrm{p}=0.000)$, which corresponded to similar studies. In bivariate Haugejorden $\mathrm{O}$ et al., reported logistic regression analyses, the odds ratio for having less than 20 natural teeth was 4.60 (95\% CI 2.36, 8.97) for respondents with the shortest education, 4.13 (95\% CI 2.53, 6.76) when gross personal income was low and 4.05 (95\% CI 2.43, 6.75) when gross adjusted family income was low. Of the control variables, age was the most important predictor of less than 20 teeth. Renata Cimoes et al., In regard to family income, a greater rate of tooth loss due to caries was found in those with incomes up to four times the minimum monthly wage (approximately US\$ 288.00) compared to those with incomes higher than that $(66.8 \%$ and $46.9 \%$, respectively), again with statistical significance $(\mathrm{P}<0.001)$. Giovanna Congiu et al., A low socioeconomic status compromises the ability of individuals to care for their health, leading to reduced resistance to oral and other diseases.

We have noticed that the higher the parents' education the less molar loss to happen. In the literature there was a positive relation as ours.
Silveira et al. found that parent education was related to molar loss. The more advanced the parents' education the less percentage of molar loss found. The lower the parent education level the less likely the child will have access to dental care which indirectly may cause tooth decay and ultimately tooth loss. Parent education correlated with unfilled cavities. The higher the education level the greater the probability decayed teeth are filled.17 NHANES data show that tooth decay affects 2.5 times as many preschoolers whose parents have less than a high school education (compared to those whose parents have greater educational attainment).

In our study there was a significant relation between molar loss, molar decay and dental insurance. Stansil et al., reported similar relation of dental insurance with teeth loss and decay. Also, Abhinav et al., they found that there is a significant relation between tooth decay and dental insurance, and an increase in the prevalence of caries among people with no dental insurance $(\mathrm{P}<0.05)$. Moreover, Jiang $\mathrm{Y}$ et al., they reported that the people without dental insurance coverage were more likely to have tooth loss. Tonya R. Stancil et al., they found that there is significant relation between tooth loss and caries in people without dental insurance and lack of missing teeth in insured population.

The reason behind the low number of first permanent molar loss and decayed that to be extracted, is due to the fact that this study was done in Dammam area which is a civilized city, and it is one of the main cities of factories and investments, so this gives us a clew on how civilization and education can play an important role in oral health status, as the results showed a big number of the adolescents parents were highly educated.

This study was done among intermediate school students, which made the use of X-rays difficult to achieve due to the lack of facilities and school law restrictions. As in Saudi Arabia the local traditions made it impossible to include female in the study to compare genders and to find differences if any. Also due to the lack of time and schools restrictions as letters should be sent to the ministry and may take 
months under processing, DMFT was difficult to calculate.

\section{Conclusion}

Several factors have been significantly related to the loss of first permanent molars among adolescents in Dammam area including SES, dental insurance, and parents education. The most common cause of tooth loss was dental caries. for this reason we recommend that early intervention and prevention of caries is mandatory to reduce caries prevalence in permanent teeth as it starts soon after first permanent molar erupts. Several measures can be taken such as feature sealants, topical fluoride application, and school-based water fluoridation.

\section{Reference}

1. Bader JD, Rozer G. Dental caries prevention: The physician's role in child oral health systemic evidence review. Rockville (MD): Agency for Healthcare Research and Quality (US); 2004 Apr.pagenumber ?

2. Batista MJ, Rihas LB, Sousa MD, Risk indicators for tooth loss in adult workers,Braz Oral Res: 2012; 26: 390-6

3. Melsen B, Terp S. The influence of extractions caries cause on the development of malocclusion and need for orthodontic treatment. Swed Dent J 1982;15:163-9.

4. Albadri S, Zaitoun H, Mcdonnell ST, Davidson LE. Extraction of first permanent molar teeth: results from three dental hospitals.Br Dent J 2007; 13;203: 408-9.

5. CorrainiP.Tooth loss prevalence and risk indicators in an isolated population of Brazil. Acta Odontol Scand. 2009;67:297303.

6. Saheeb BD, Sede MA. Reasons and pattern of tooth mortality in a Nigerian Urban teaching hospital. B Ann Afr Med 2013;12:110-4.

7. Koray $\mathrm{H}$, Orcun $\mathrm{T}$, Ismail A, Mevlut C. Permanent first molar extraction in adolescents and young adults and its effect on the development of third molar. Clin Oral Invest 2013; Volume number 2 pages 232

8. ArtunJ,ThalibL.Mesial migration and loss of first molars among young adolescents in Kuwait. Community Dent Health. 2011;28:154-9

9. Khalil A, Hesham SK. Reasons for and patterns relating to the Extraction of permanent teeth in a subset of the Saudi population. ClinCosmetInvestig Dent. 2013; 30:5:51-6.

10. The General Administration of Edcuation in the Eastern province, http://www.edueast.gov.sa/portal/232

11. Raosoft, sample size calculator. http://www.raosoft.com/samplesize.html

12. AtiehMA.Tooth loss among Saudi adolescents: social and behavioural risk factors, Int Dent J. 2008 Apr;58(2):103-8.

13. Haugejorden $\mathrm{O}$, Klock KS, $\mathrm{A}^{\circ} \operatorname{str} \varnothing \mathrm{m}$ AN, Skaret E, Trovik TA. Socio-economic inequality in the self-reported number of natural teeth among Norwegian adults - an analytical study. Community Dent Oral Epidemiol 2008; 36: 269-278.

14. Renata C, Arnaldo de, Primary Reason for Tooth Extraction in a Brazilian Adult Population,oral health prev Dent, 2005;3(3):151-7

15. Giovanna C, Guglielmo C, Silvana S, Early childhood caries and associated determinants, Journal of Public Health Dentistry, 2013 Oct 9.

16. Silveira RC, Primary reason for tooth extraction in a Brazilian adult population, Oral Health Prev Dent. 2005;3(3):151-7.

17. Edelstein BL, Disparities in Oral Health and Access to Care: Findings of National Surveys, Ambul Pediatr. 2002 Mar-Apr;2(2 Suppl):141-7

18. Vargas CM, Crall JJ, Schneider DA. Sociodemographic distribution of pediatric dental caries: NHANES III, 1988-1994 J Am Dent Assoc. 1998;129:1229-1288 
19. Abhinav S, Bharathi M, Nitin M, Risk factors for oral diseases among workers with and without dental insurance in a national social security scheme in India, International Dental Journal 2014; 64: 8995.

20. YongwenJ,sociodemographic and health related risk factors associated with tooth loss, Prev Chronic Dis 2013:10.

21. Tonya R, Jeffrey J, Dental Insurance and Clinical Dental Outcomes in NHANES III,J public health Dent, 2005 Fall. 